\title{
Efficacy of celecoxib in the treatment of CNS lymphomas: an in vivo model
}

\author{
Weijun Wang, M.D., Adel Kardosh, M.S., Yuzhuang S. Su, M.S., \\ Axel H. Schonthal, Ph.D., And Thomas C. Chen, M.D., Ph.D.
}

Departments of Neurosurgery, Molecular Microbiology \& Immunology, and Pathology, University of Southern California, Los Angeles, California

\begin{abstract}
Object. The incidence of primary central nervous system lymphomas (PCNSLs) has increased over the past several decades. Unfortunately, even with the most effective therapeutic regimen (that is, methotrexate with wholebrain radiation therapy), PCNSL recurs within a few years in more than half of the treated patients and is eventually fatal. Because PCNSL usually occurs in older patients and in those with acquired immunodeficiency syndrome, combination treatments in which both chemo- and radiation therapy are used is often poorly tolerated and results in a significant reduction in the quality of life. Recently, it has been demonstrated that the selective cyclooxygenase2 inhibitor celecoxib (Celebrex), can block the growth of lymphoma cells in vitro.

Methods. To create an experimental animal model in vivo for the PCNSL study, the authors intracranially injected a human B-cell lymphoma cell line into nude mice. Their data demonstrate that this experimental model is an excellent one for human PCNSL with brain and leptomeningeal involvement. They also evaluated the feasibility of using celecoxib as a therapeutic agent in the treatment of PCNSL. Nude mice with intracranial lymphomas were treated with celecoxib contained in the animal chow. The treated animals demonstrated significantly prolonged survival times compared with the untreated animals.

Conclusions. Based on the authors' data, celecoxib may be a promising therapeutic agent for the treatment of PCNSL.
\end{abstract}

KEY WORDS $\bullet$ cyclooxygenase-2 inhibitor $\bullet$ celecoxib $\bullet$
primary central nervous system lymphoma $\bullet$ chemotherapy $\bullet$ mouse

$\mathrm{P}$

RIMARY CNS lymphomas account for 1 to $2 \%$ of all non-Hodgkin lymphomas and for 2 to $7 \%$ of all primary CNS tumors. ${ }^{6,17}$ These tumors can involve the brain, spinal cord, meninges, or orbit. The incidence of PCNSL has increased threefold since the $1970 \mathrm{~s} ; ;^{10}$ most cases involve non-Hodgkin B-cell lymphomas. ${ }^{5}$ In contrast, Hodgkin disease rarely involves the CNS. ${ }^{4}$

The survival time for patients with a PCNSL who do not undergo treatment is approximately 3 months after diagnosis. Even with treatment, the prognosis of PCNSL is still poor. After whole-brain radiation therapy alone is performed, the 2-year overall survival rate is $28 \%$. The survival rate can be improved by adding high-dose methotrexate-based chemotherapy in conjunction with whole brain-radiation therapy. ${ }^{1,3}$ Using this regimen, a 2year survival rate of 50 to $70 \%$ 7,21,22 and a 5-year survival

Abbreviations used in this paper: $\mathrm{CNS}=$ central nervous system; COX-2 = cyclooxygenase- 2 ; DMC = derivative dimethyl celecoxib; IACUC = Institutional Animal Care and Use Committee; MTT = 3-(4,5-dimethylthiazol-2-yl)2,5-diphenyltetrazolium bromide; NSAID $=$ nonsteroidal antiiflammatory drug; $\mathrm{PBS}=$ phosphate-buffered saline; PCNSL = primary CNS lymphoma; TUNEL = terminal deoxynucleotidyl transferase (TdT)-mediated deoxyuridine triphosphate (dUTP) nick-end labeling technique; USC $=$ University of Southern California. rate greater than $30 \%$ can be achieved. ${ }^{8}$

However, late neurotoxicity develops in up to $80 \%$ of the patients after combined therapy, and it is attributable to both the disease burden and the deleterious effects of treatment, including dementia, gait ataxia, and urinary dysfunction..$^{1,2}$

Recently, we demonstrated that the COX-2 selective inhibitor celecoxib, a well-tolerated oral NSAID, has antiproliferative properties in different lymphoma cell lines (Raji and Ramos Burkitt lymphomas) in vitro. ${ }^{14}$ In this paper, we describe an in vivo intracranial lymphoma model with leptomeningeal metastasis mimicking human CNS lymphoma. Our in vivo data demonstrate that the lymphoma cells disseminate within the brain (in the cerebral cortex and periventricular region) as well as in the leptomeninges. The therapeutic effect of celecoxib in vivo was tested in this animal model, and the survival data were plotted according to Kaplan-Meier methods.

\section{Materials and Methods}

\section{Lymphoma Cell Cultures}

Three human cell lines were used: Burkitt lymphoma cell lines (Raji and Ramos, American Tissue Culture Collection, Manassas, VA) and one primary cell line (A6876) from a patient with non-Hodgkin lymphoma (kindly pro- 
W. Wang, A. Kardosh, and Y. S. Su, et al.

vided by Dr. Parkash S. Gill; University of Southern California, Los Angeles, CA). ${ }^{16}$ All cells were grown in suspension and propagated in RPMI 164 (Gibco BRL, Grand Island, NY) supplemented with $10 \%$ fetal bovine serum, $50 \mathrm{U} / \mathrm{ml}$ penicillin, and $50 \mu \mathrm{g} / \mathrm{ml}$ streptomycin at $37^{\circ} \mathrm{C}$ with a humidified atmosphere of $5 \% \mathrm{CO}_{2}$. The medium was replaced every 3rd day after cell centrifugation at 800 rpm for 3 minutes.

\section{The MTT assay}

The MTT assay was used as a cytotoxicity assay for the Ramos, Raji, and A6876 cells using celecoxib. Briefly, cells $\left(2 \times 10^{5}\right.$ cells $\left./ \mathrm{ml}\right)$ were seeded into 96 -well plates and treated with varying concentrations of celecoxib $(0-500 \mu \mathrm{M})$ for 48 hours. The MTT assay was performed using a commercially available MTT assay kit (Roche Diagnostics, Indianapolis, IN) according to manufacturer's instructions. The remaining viable cells with MTT dye uptake were determined by measuring the optical density at $490 \mathrm{~nm}$ in an enzyme-linked immunosorbent assay reader. Values shown are the mean \pm standard deviation of at least six measurements. ${ }^{14}$

\section{Animal Model of Intracranial CNS Lymphoma}

All animal protocols were approved by the IACUC of USC. All mice were maintained in a pathogen-free environment throughout the experiment. Briefly, athymic mice (Harlan, Inc., Indianapolis, IN) were anesthetized with an intraperitoneal injection of $80 \mathrm{mg} / \mathrm{kg}$ of ketamine and xylazine using a dose of $2 \mathrm{mg} / \mathrm{kg}$ body weight. Animals were fixed into the stereotactic headframe, a paramedian incision was made, and a 1.5-mm bur hole was drilled $1 \mathrm{~mm}$ anterior to the coronal suture on the right hemisphere and $2 \mathrm{~mm}$ lateral from the midline. A Hamilton syringe was used to inject the Raji lymphoma cells $\left(5 \times 10^{5}\right.$ cells/ $10 \mu \mathrm{l}$ ) into the right frontal lobe of the brain. The skin incision was closed with silk thread.

\section{Celecoxib Treatment Strategy}

Ten mice were divided into two groups (five animals per group). All mice were implanted stereotactically with $5 \times 10^{5}$ Raji cells as described earlier. The day after tumor cell implantation, five mice commenced treatment by consuming chow containing celecoxib (1000 ppm). The other group consumed fresh chow only. To make the celecoxib chow, fresh chow powder was reconstituted with PBS and $1000 \mathrm{ppm}$ celecoxib. Semisolid rectangular blocks $(2 \times 3$ $\times 0.5 \mathrm{~cm}$ ) were made and baked in a $100^{\circ} \mathrm{C}$ oven; the vacuum was turned on overnight to dry up and harden the food before the mice were fed.

\section{Survival Analysis}

Animals were killed at the end point set forth according to the protocol submitted to the IACUC of USC. The survival of animals with intracranial lymphoma treated with or without celecoxib was plotted using Kaplan-Meier curves. The survival difference between the two groups was compared, and statistical significance was determined by the paired Student t-test; a probability value less than 0.05 was considered to be statistically significant.

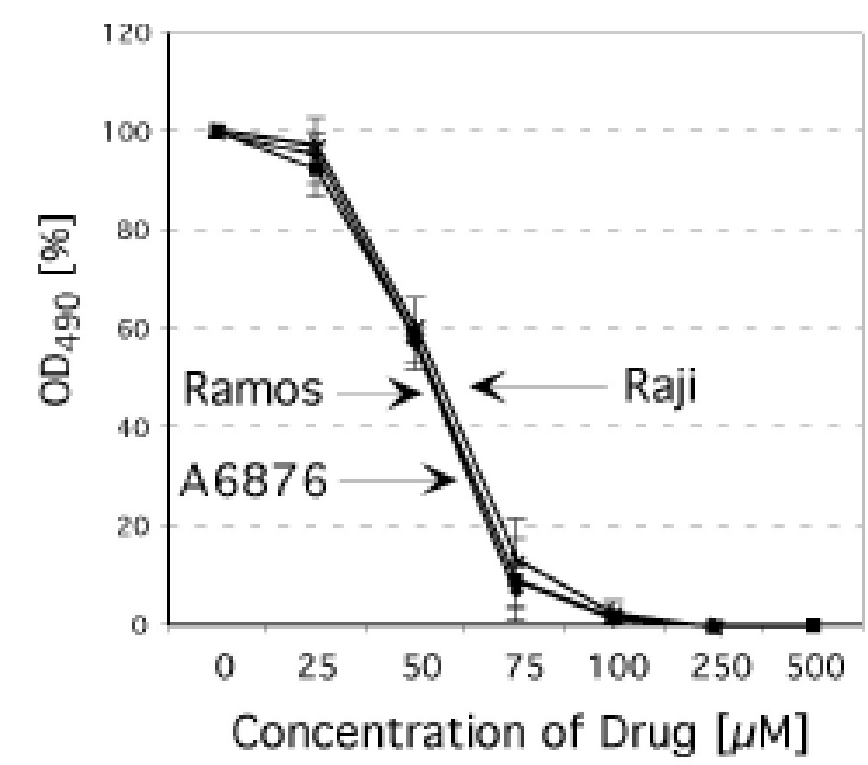

FIG. 1. Graph showing the cytotoxicity assay after celecoxib treatment of lymphoma cells in vitro. The effect of celecoxib on the cytotoxicity of Raji and Ramos Burkitt lymphoma cells and A6876 non-Hodgkin lymphoma cells was analyzed with a commercially available MTT assay kit (Roche Diagnostics, Indianapolis, IN). Cells were seeded at $2 \times 10^{5}$ cells $/ \mathrm{ml}$ in 96 -well plates and supplemented with varying doses of celecoxib to yield the respective final concentrations $(0-500 \mu \mathrm{M})$ as indicated. Forty-eight hours later, MTT dye was added, and the cells were incubated for another 4 hours before the reaction was terminated. Cell viability was determined by measuring the optical density (OD) at $490 \mathrm{~nm}$ in an enzyme-linked immunosorbent assay plate reader. Values shown are the mean \pm standard deviation of at least six measurements.

\section{Immunohistochemical Analysis}

When mice displayed focal neurological deficits, such as the failure to extend their paws fully, failure to turn to one side, or circling on one side, they were killed according to USC IACUC guidelines. After they were killed, the brain, cervical, and thoracic vertebrae were removed, embedded in optimal cutting medium, and stored at $-800^{\circ} \mathrm{C}$ for future analysis. Brain and vertebrae with spinal cord were sectioned serially for immunohistochemical analysis. Sections were stained with $\mathrm{H} \& \mathrm{E}$ to detect human lymphoma cells inside the brain, spinal cord, and leptomeninges. For the immunohistochemical analysis, sections were fixed in acetone for 5 minutes, dried for 10 minutes, quenched in $0.3 \% \mathrm{H}_{2} \mathrm{O}_{2}$ for 5 minutes, and washed three times in cold PBS. Nonspecific binding was preblocked for 30 minutes at room temperature. Sections were incubated overnight at $4{ }^{\circ} \mathrm{C}$ with a 1:200 dilution of the following primary antibodies: bcl-2 and survivin (Santa Cruz Biotechnology, Inc., Santa Cruz, CA). Slides were then incubated with a secondary antibody at a 1:200 dilution at room temperature after three washes in PBS. Immunoreactivity was visualized with a VECTASTAIN $\mathrm{ABC}$ kit (Vector Laboratories, Burlingame, CA), using 3amino-9-ethylcarbazole as a chromogen, and they were counterstained with hematoxylin. The B-cell marker (CD20), common leukocyte antigen (CD45), and a T-cell marker (CD3) were obtained from Santa Cruz Biotechnology, Inc., and used to identify the xenografts. 


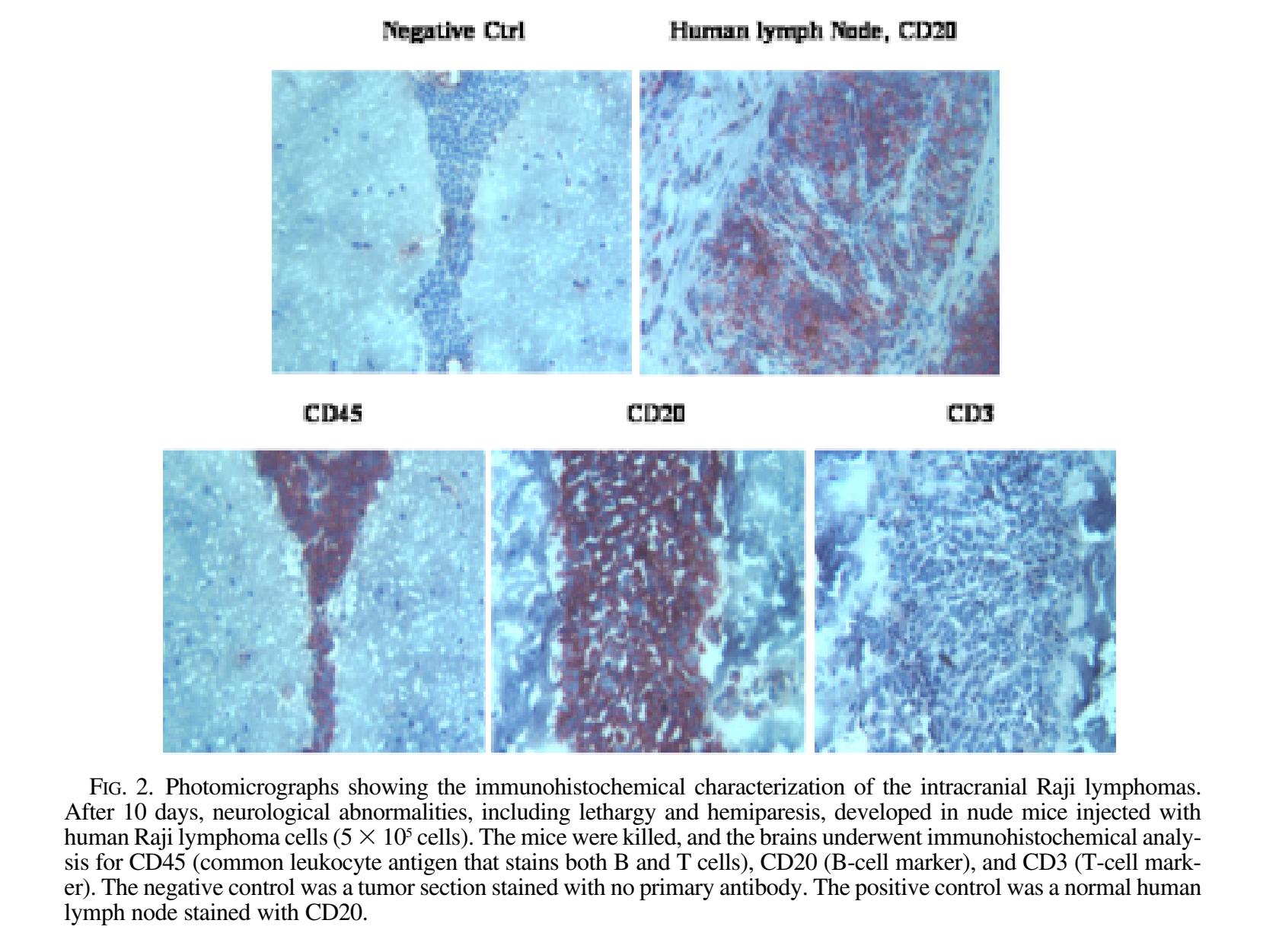

\section{The TUNEL Assay}

The technique was used to detect $3^{\prime}-\mathrm{OH}$ ends of the DNA exposed during the internucleosomal cleavage process of apoptosis. The assay was performed using the ApopTag apoptosis detection kit (Invitrogen, New York, NY) according to the manufacturer's protocol.

\section{Results}

Celecoxib Induces Cytotoxicity on Lymphoma Cells In Vitro

To investigate whether NSAIDs could be considered for the new treatment strategies for lymphomas, we determined the effects of celecoxib on three different lymphoma cell lines in vitro: Raji, Ramos, and A6876. Increasing concentrations of celecoxib $(0-500 \mu \mathrm{M})$ were added, and cytotoxicity was determined using MTT assay. As shown in Fig. 1, celecoxib demonstrated a potent inhibition of all three lymphoma cell lines in vitro, with a median drug concentration for inhibitory effect in the range of 55 to $75 \mu \mathrm{M}$ for all three cell lines examined.

\section{Validation of the CNS Lymphoma Model}

Mice were implanted intracerebrally with Raji lymphoma cells and killed 10 days postimplantation. A microscopic analysis demonstrated the formation of tumor intracranially, with leptomeningeal involvement. An im- munohistochemical analysis demonstrated that these lymphoma cells were strongly positive for CD45 (common leukocyte antigen, staining both $\mathrm{B}$ and T cells) and CD20 (B cells) but were negative for the T-cell marker CD3 (Fig. 2). Staining with $\mathrm{H} \& \mathrm{E}$ was used for the detection of lymphomatous meningitis. Figure 3 demonstrates lymphoma cells in the brain parenchyma (Fig. 3A), with perivascular infiltration and cuffing (Fig. 3B). Confirmation that these cells were B-cell lymphomas was confirmed by positive immunostaining for CD20 and CD45 but negative immunostaining for CD3. Lymphoma cells were also seen in the leptomeninges of the cerebellum, with infiltration into the cerebellar parenchyma; periventricular involvement was also identified (data not shown). In addition to cerebral involvement, spinal cord and leptomeningeal invasion can be clearly demonstrated as shown in Fig. 4A and B. The B-cell lymphomas (CD20 and CD45 positive; CD3 negative) were clearly seen in the leptomeninges but not in the spinal cord parenchyma. Our data demonstrate that this is an excellent animal model to study human PCNSL, mimicking the brain and leptomeningeal involvement in human PCNSL.

\section{Celecoxib Induces Apoptosis of Lymphomas In Vivo}

To determine whether the cytotoxic effects of celecoxib determined on MTT assay (Fig. 1) are secondary to apoptosis, TUNEL assay was used. Figure 5 demonstrates that there are more apoptotic cells in the celecoxib-treated 


\section{Brain parenchyma}

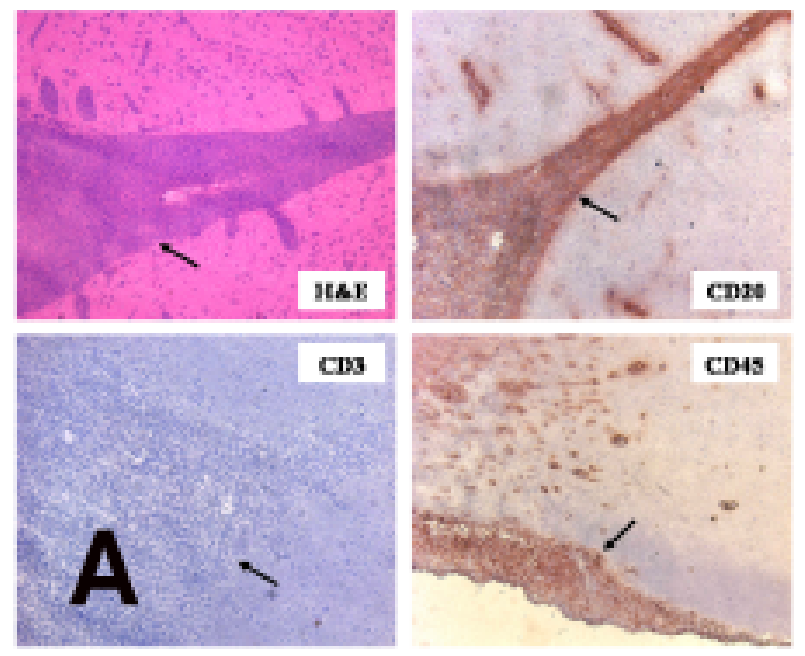

Brain perivascular cuffing

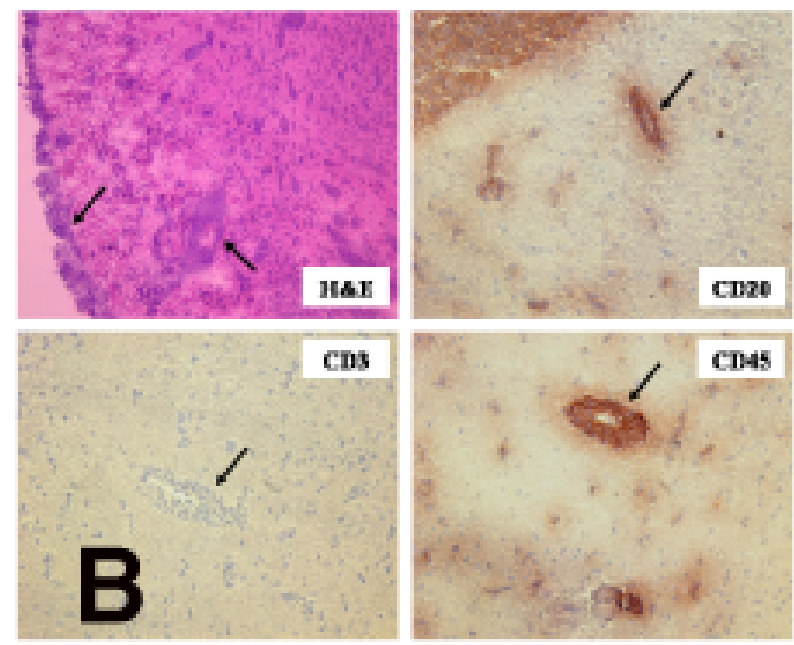

FIG. 3. Photomicrographs of brain parenchyma after Raji lymphoma implantation. The mice were killed after neurological deficits developed. The brain was removed and sectioned serially for immunohistochemical analysis. A: Lymphoma cells infiltrate into the normal brain parenchyma and are present in the cortex and leptomeninges. B: Perivascular cuffing typical of lymphomas is seen. Immunostaining was performed to confirm that cells were CD20 and CD45 positive, but CD3 negative. Original magnification $\times 10(\mathrm{a}) ; \times 20(\mathrm{~b})$.

lymphomas compared with the PBS-treated tumors. Moreover, there was a decrease in the expression of the antiapoptotic protein bcl-2 (Fig. 5), with no attendant increase in the proapoptotic protein bax (data not shown). The same results were detected for the lymphomas in the leptomeninges (data not shown). We also examined the effects of celecoxib on the antiapoptotic protein survivin. We had previously demonstrated that celecoxib decreased the survivin protein in glioma cells. ${ }^{15}$ Figure 5 demonstrates that survivin was significantly downregulated after treatment with celecoxib.

\section{Celecoxib Prolongs Survival of Mice With Intracranial Lymphomas}

Improvement in the survival duration of mice with CNS lymphomas treated with celecoxib is demonstrated on a Kaplan-Meier survival curve (Fig. 6). Animal survival was compared between the celecoxib- and PBS-treated mice. The difference of prolonged survival time was calculated using the paired Student t-test $(\mathrm{p}<0.05$ considered significant). Our data demonstrated that the animals treated with celecoxib had significantly prolonged survival times compared with the PBS-treated mice. The celecoxib-treated mice survived up to 9 days longer than those treated with PBS alone.

\section{Discussion}

We have previously demonstrated that celecoxib and its non-COX-2 DMC can inhibit Burkitt lymphoma cells. ${ }^{14}$ Traditional NSAIDs have been demonstrated to block proliferation in vitro and in vivo of certain human tumors, such as colon and lung carcinoma. ${ }^{9,13}$ In our previous study we demonstrated the greatest efficacy with celecoxib and DMC, as opposed to other NSAIDs such as sulindac, indomethacin, valdecoxib (Vioxx), and rofecoxib (Bextra). ${ }^{14}$ Wun, et al., ${ }^{26}$ have demonstrated that COX-2 is increased by 2 to 2.3 times in primary B-cell lymphoma. However, our previous data demonstrating that celecoxib is superior to valdecoxib and rofecoxib suggest that more than $\mathrm{COX}$ 2 inhibition is involved. These data are further supported by the fact that DMC, which does not inhibit COX-2, is also cytotoxic to lymphoma cells. An increased understanding of the pharmaceutical action of celecoxib may enable development of more targeted treatment of lymphomas in the future.

In this study, we have established an intracranial CNS lymphoma model with leptomeningeal metastasis using Raji Burkitt lymphoma cells. Although we had demonstrated in vitro (via MTT assay) that Celebrex was not only effective against Raji cells, but also against Ramos and A6876 lymphoma cells, the Raji cell line was used because it had better tumor uptake in nude mice than the Ramos cell line. We did not use the A6876 primary cell line because it was not as well characterized as the Raji and Ramos cell lines. Using the Raji cell line, we have demonstrated that celecoxib is effective in inhibiting CNS lymphoma growth in vivo. Other CNS lymphoma models have been previously established. Saini, et al., ${ }^{24}$ used human B lymphoma cells (BL2) and implanted them into New Zealand nude rats; an $88 \%$ uptake was noted, with diffuse parenchymal and periventricular spread. No extracerebral, spinal, or cerebellar metastasis was seen with this model. More recently, Soussain, et al. ${ }^{25}$ have described a nude rat model in which MC116 human Blymphoma cells were implanted into the caudate nucleus; infiltrative lymphoma cells were seen in the cortex, meninges, and ventricles. Rats were treated with radiation therapy or methotrexate, and MR images were obtained pre- and posttreatment. The closest model to our Raji lymphoma model was that established by Roychowdhury, et al., ${ }^{23}$ who implanted Epstein-Barr virus-transformed lymphoblasts into the caudate nucleus of nude rats. The authors noted the onset of focal neurological symptoms within 21 days. 

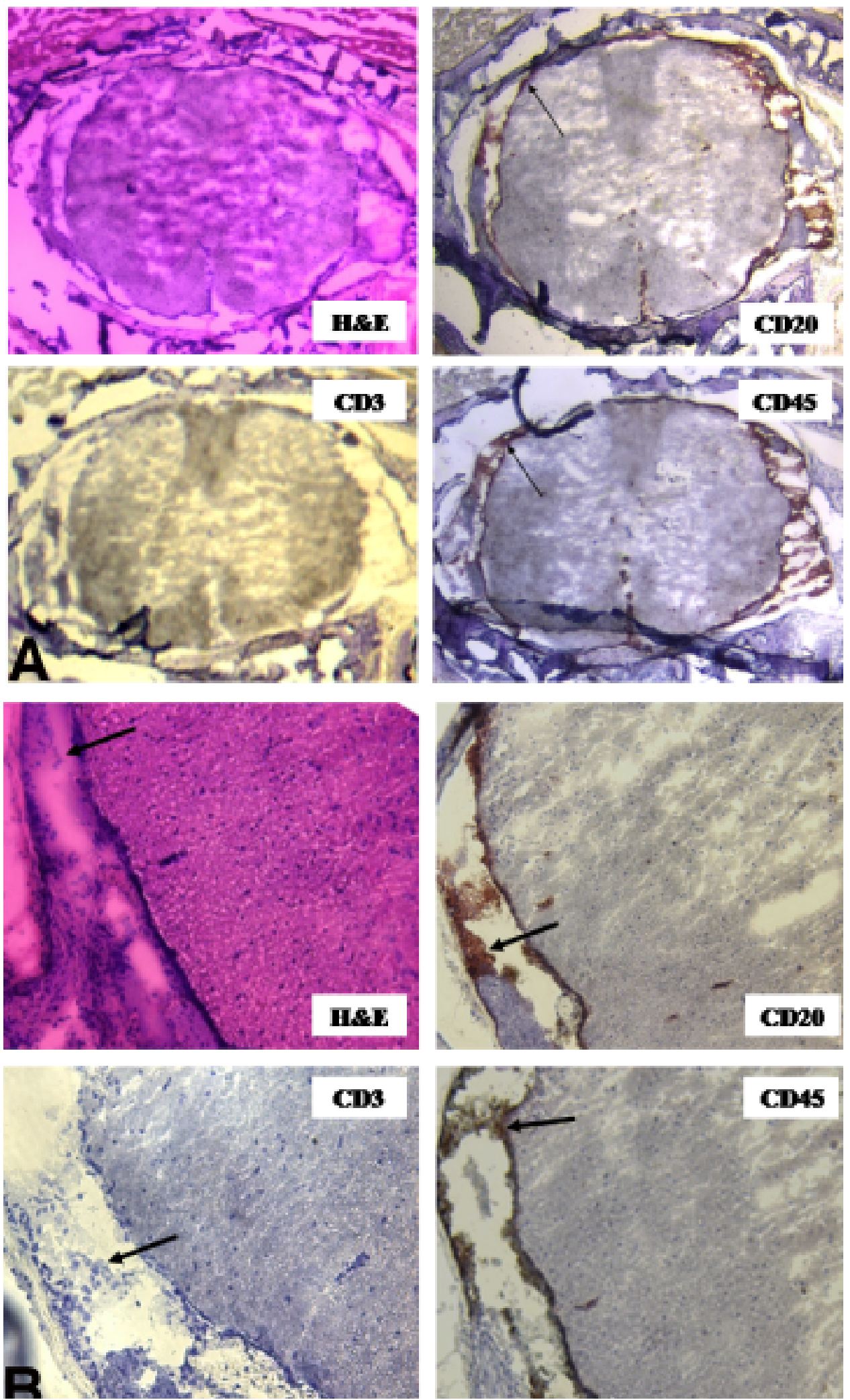

FIG. 4. Photomicrographs of the leptomeninges and spinal cord. Mice injected intracranially with Raji lymphoma cells were killed after neurological deficits developed. Vertebrae and spinal cord were harvested simultaneously and sectioned serially for immunohistochemical analysis. A: Low-power magnification view of thoracic spinal cord, demonstrating CD20- and CD45-positive lymphoma cells in the leptomeninges. B: High-power magnification of Raji lymphoma cells (CD20 and CD45 positive; CD3 negative) in the leptomeninges of the thoracic spinal cord with subarachnoid invasion. Original magnification $\times 2.5(\mathrm{~A}) ; \times 20(\mathrm{~B})$. 

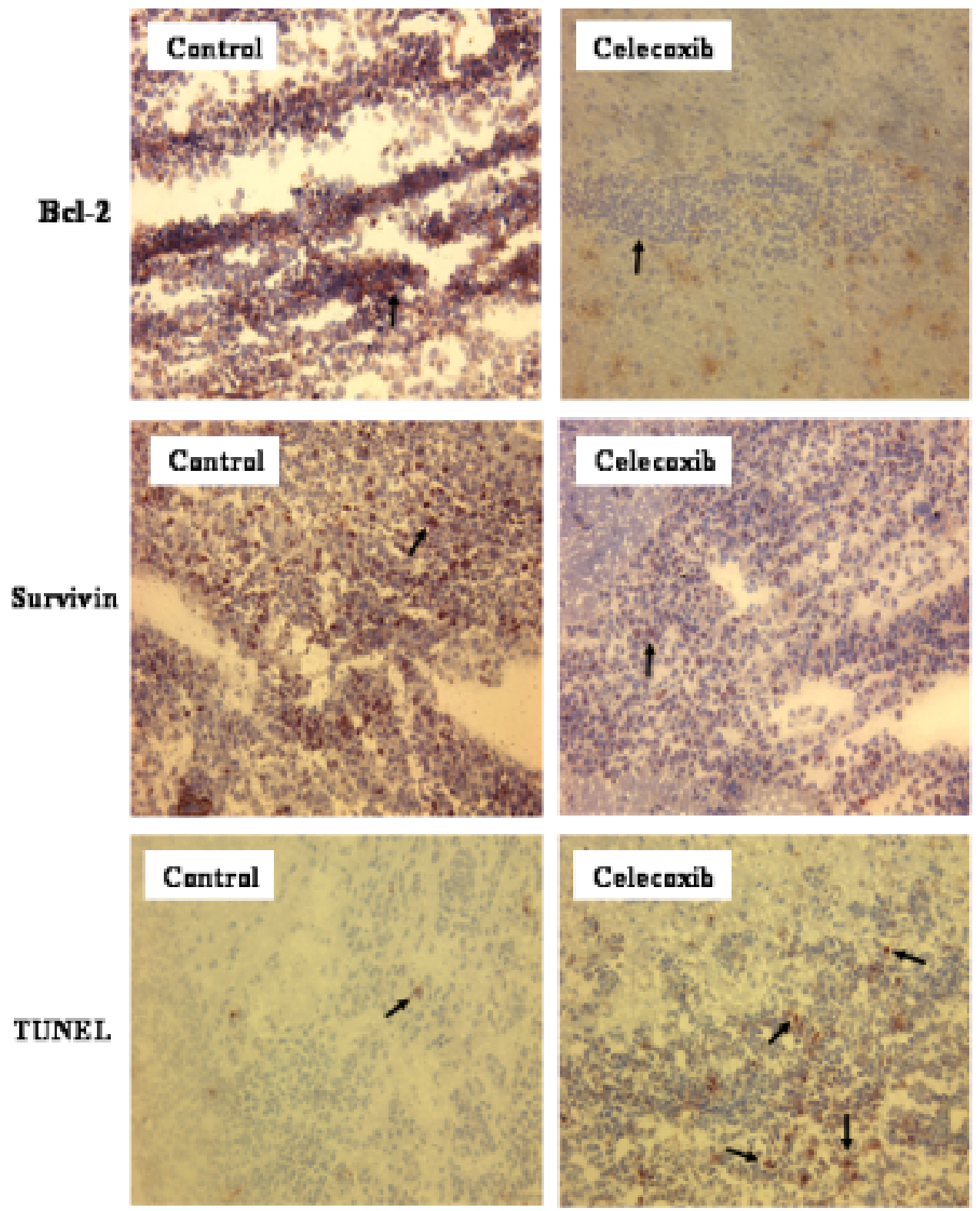

Fig. 5. Photomicrographs. Analysis for apoptosis and the antiapoptotic proteins bcl-2 and survivin before and after treatment with celcoxib. Mice implanted intracranially with Raji cells were divided into two groups (five mice/group): control (no celecoxib) and celecoxib (1000 ppm chow). After the mice were killed, the brains were harvested and sectioned serially for immunohistochemical analysis. Lymphoma cells from control mice had high levels of the antiapoptotic proteins bcl-2 and survivin; TUNEL staining for apoptosis was minimal. Lymphoma cells from celecoxib-treated mice had decreased levels of bcl-2 and survivin, with an increased number of positive apoptotic cells (arrows).

Our CNS lymphoma model does mimic PCNSL in humans. We have demonstrated lymphoma cells via CD20 and CD45 staining in the brain parenchyma and leptomeninges. In the brain parenchyma, periventricular involvement and perivascular cuffing can be visualized, similar to PCNSL in humans. We did not focus on ocular involvement or cerebrospinal fluid involvement in the killed mice; these studies are currently ongoing. Unlike other published CNS lymphoma models, to our knowledge this model is the first one in nude mice. Nude mice have the advantage of being cheaper to use and easier to handle than rats; however, imaging studies are much hard- er to obtain. Our model does have the limitation, however, of using Burkitt lymphoma cells, which are usually associated with HIV and Epstein-Barr virus infections. Burkitt lymphomas in the CNS usually occur in HIV-positive patients and are often metastatic. ${ }^{19}$ Although Burkitt lymphomas have been reported for PCNSL in immunocompetent patients, they are extremely rare. ${ }^{11,12,15,18} \mathrm{We}$ chose the Raji cell line because we had previously demonstrated in vitro the potent cytotoxic effect of celecoxib on it. Although celecoxib also had very cytotoxic effects on the other Burkitt lymphoma cell lines (Ramos) and A6876, these cell lines were not tested in this particular 


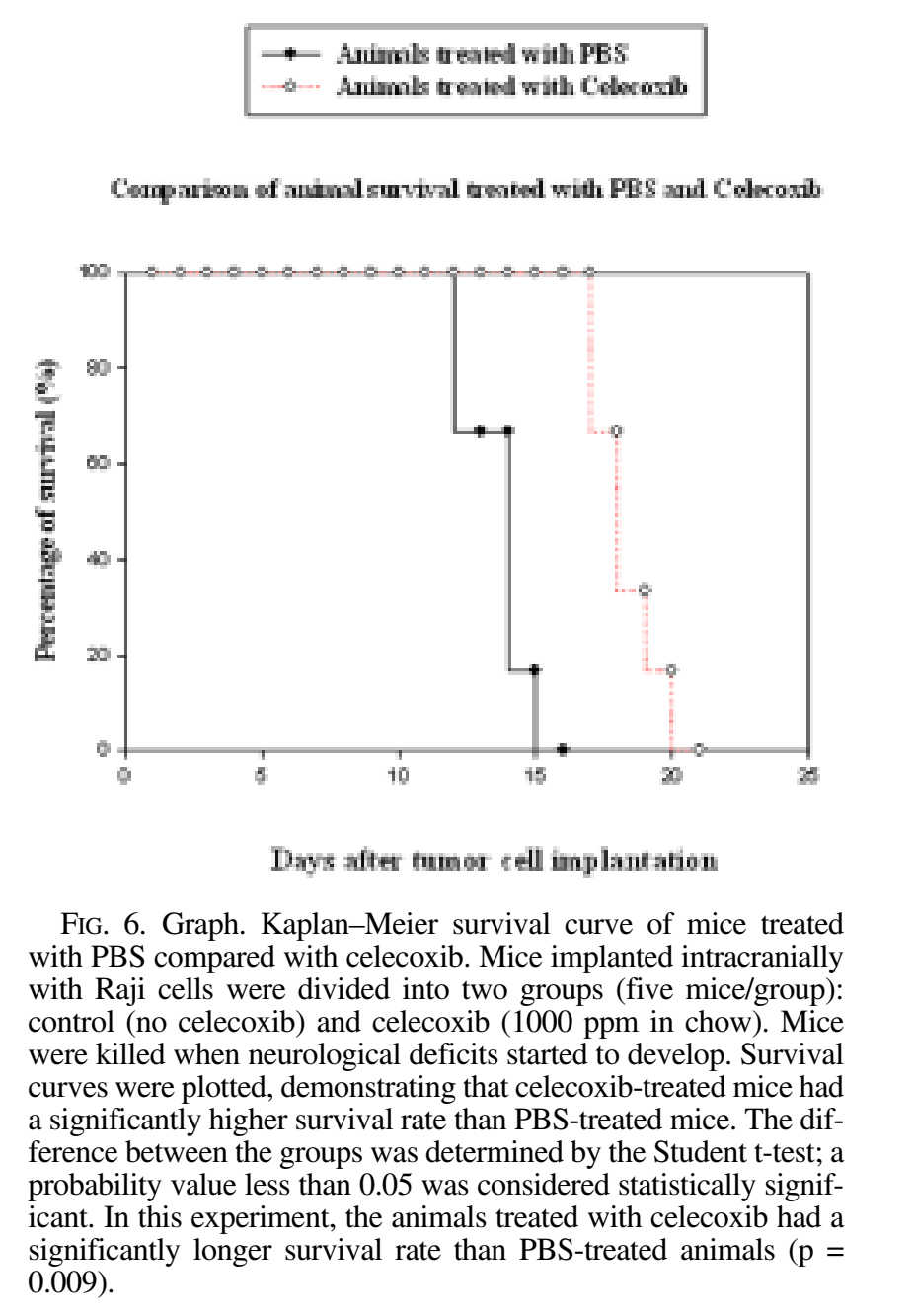

study against celecoxib in vivo because of variation in their tumorgenicity in athymic nu/nu mice. Our expectation, however, is that the Ramos and A6876 lymphoma cells, once established in an in vivo model, would yield similar results.

Besides demonstrating that celecoxib had a cytotoxic effect on intracranial Raji lymphomas, we also demonstrated that the mechanism of the cytotoxicity was via induction of apoptosis. The mechanism of apoptosis is in part secondary to the decreased bcl-2 and survivin expression in the treated tumors, compared with the nontreated tumors. Celecoxib was also able to substantially reduce bcl-2 and survivin expression in the Raji tumor cells that had infiltrated into the leptomeninges, indicating good generalized CNS penetrance.

Our particular in vivo animal study, however, does highlight the potential disadvantages of using oral medications in a fast-growing tumor. Celecoxib was baked into the animal chow and ingested orally on a daily basis. However, when the intracranial pressure increased, food intake by the mice decreased greatly as they became more lethargic. One of the ways the survival advantage with oral celecoxib can be enhanced is by ensuring its intake as the animals became more impaired due to their intracranial disease. In the future, we will deliver celecoxib by oral lavage. By using a 24-gauge round-tipped feeding needle (Pepper \& Sons, Inc., New Hyde Park, New York), the same dosage of celecoxib will be directly delivered to the stomach. Theoretically, the animal survival rate should improve with better delivery.

It has also been reported that celecoxib can function as a radiation therapy and chemotherapy sensitizer. ${ }^{20}$ Future experiments will concentrate on combining celecoxib with methotrexate chemotherapy or radiation therapy. If successful, combination treatment with celecoxib may be used to decrease the toxicity of methotrexate and/or radiation therapy. Moreover, celecoxib may potentially be used on a chronic basis to decrease lymphoma recurrence. The addition of celecoxib may improve the current management and quality of life of these devastated patients.

\section{References}

1. Abrey LE, DeAngelis LM, Yahalom J: Long-term survival in primary CNS lymphoma. J Clin Oncol 16:859-863, 1998

2. Abrey LE, Yahalom J, DeAngelis LM: Treatment for primary CNS lymphoma: the next step. Clin Oncol 18:3144-3150, 2000

3. Correa DD, DeAngelis LM, Shi W, Thaler H, Glass A, Abrey LE: Cognitive functions in survivors of primary central nervous system lymphoma. Neurology 62:548-555, 2004

4. Davenport RD, O'Donnell LR, Schnitzer B, McKeever PE: NonHodgkin's lymphoma of the brain after Hodgkin's disease: an immunohistochemical study. Cancer 67:440-443, 1991

5. DeAngelis LM: Primary central nervous system lymphoma. J Neurol Neurosurg Psychiatry 66:699-701, 1999 (Editorial)

6. DeAngelis LM: Primary CNS lymphoma: treatment with combined chemotherapy and radiotherapy. J Neurooncol 43: 249-257, 1999

7. DeAngelis LM, Seiferheld W, Schold SC, Fisher B, Schultz CJ: Combination chemotherapy and radiotherapy for primary central nervous system lymphoma: radiation therapy oncology group study 93-10. J Clin Oncol 20:4643-4648, 2002

8. DeAngelis LM, Yahalom J, Heinemann MH, Cirrincione C, Thaler HT, Krol G: Primary CNS lymphoma: combined treatment with chemotherapy and radiotherapy. Neurology 40: 80-86, 1990

9. Duffy CP, Elliott CJ, O'Connor RA, Heenan MM, Coyle S, Cleary IM, et al: Enhancement of chemotherapeutic drug toxicity to human tumor cells in vitro by a subset of non-steroidal anti-inflammatory drugs (NSAIDs). Eur J Cancer 34: 1250-1259, 1998

10. Eby NL, Grufferman S, Flannelly CM, Schold SC Jr, Vogel FS, Burger PC: Increasing incidence of primary brain lymphoma in the US. Cancer 62:2461-2465, 1988

11. Giromini D, Peiffer J, Tzonos T: Occurrence of a primary Burkitt-type lymphoma of the central nervous system in an astrocytoma patient. A case report. Acta Neuropathol 54: $165-167,1981$

12. Hegedus K: Burkitt-type lymphoma and reticulum-cell sarcoma. An unusual mixed form of two intracranial primary malignant lymphomas. Surg Neurol 21:23-29, 1984

13. Hida T, Kozaki K, Ito H, Miyaishi O, Tatematsu Y, Suzuki T, et al: Significant growth inhibition of human lung cancer cells both in vitro and in vivo by the combined use of a selective cyclooxygenase 2 inhibitor, JTE-522, and conventional anticancer agents. Clin Cancer Res 8:2443-2447, 2002

14. Kardosh A, Wang W, Uddin J, Petasis NA, Hofman FM, Chen TC, et al: Dimethyl-celecoxib (DMC), a derivative of celecoxib that lacks cyclooxygenase-2-inhibitory function, potently mimics the anti-tumor effects of celecoxib on Burkitt's lymphoma in vitro and in vivo. Cancer Biol Ther 4:571-582, 2005 
15. Kobayashi H, Sano I, Ii K, Hizawa K: Primary Burkitt-type lymphoma of the central nervous system. Acta Neuropathol 64:12-14, 1984

16. Masood R, McGarvey ME, Zheng T, Cai J, Arora N, Smith DL, et al: Antineoplastic urinary protein inhibits Kaposi's sarcoma and angiogenesis in vitro and in vivo. Blood 93:1038-1044, 1999

17. Miller DC, Hochberg FH, Harris NL, Gruber ML, Louis DN, Cohen H: Pathology with clinical correlations of primary central nervous system non-Hodgkin's lymphoma: the Massachusetts General Hospital experience 1958-1989. Cancer 74:1383-1397, 1994

18. Monabati A, Rakei SM, Kumar P, Taghipoor M, Rahimi A: Primary Burkitt lymphoma of the brain in an immunocompetent patient. Case report. J Neurosurg 96:1127-1129, 2002

19. Mwanda OW: Aspects of epidemiological and clinical features of patients with central nervous system Burkitt's lymphoma in Kenya. East Afr Med J 81 (Suppl): S97-S103, 2004

20. Nieder C, Grosu AL, Astner S, Thamm R, Molls M: Integration of chemotherapy into current treatment strategies for brain metastases from solid tumors. RadiatOncol 1:19, 2006

21. O'Brien P, Roos D, Pratt G, Liew K, Barton M, Poulsen M, et al: Phase II multicenter study of brief single-agent methotrexate followed by irradiation in primary CNS lymphoma. J Clin Oncol 18:519-526, 2000

22. Poortmans PM, Kluin-Nelemans HC, Haaxma-Reiche H, Van't Veer M, Hansen M, Soubeyran P, et al: High-dose methotrexate-based chemotherapy followed by consolidating radiotherapy in non-AIDS-related primary central nervous system lym-
W. Wang, A. Kardosh, and Y. S. Su, et al.

phoma. European Organization for Research and Treatment of Cancer Lymphoma Group Phase II Trial 20962. J Clin Oncol 21:4483-4488, 2003

23. Roychowdhury S, Peng R, Baiocchi RA, Bhatt D, Vourganti S, Grecula J, et al: Experimental treatment of Epstein-Barr virusassociated primary central nervous system lymphoma. Cancer Res 63:965-971, 2003

24. Saini M, Bellinzona M, Weichhold W, Samii M: A new xenograft model of primary central nervous system lymphoma. J Neurooncol 43:153-160, 1999

25. Soussain C, Muldoon LL, Varallyay C, Jahnke K, Neuwelt EA: Characterization and magnetic resonance imaging of a rat model of human B cell central nervous system lymphomas: potential for assessing therapy and neurotoxicity. Proc Am Assoc Cancer Res 47:645-646, 2006 (Abstract)

26. Wun T, McKnight H, Tuscano JM: Increased cyclooxygenase2 (COX-2): a potential role in the pathogenesis of lymphoma. Leukemia Res 28:179-190, 2004

This work was supported by awards from the Harry and Marion Kieper Fund for Neurosurgical Research and the Sounder Foundation.

Manuscript received August 30, 2006.

Accepted in final form October 4, 2006.

Address reprint requests to: Thomas C. Chen, M.D., Ph.D., Department of Neurosurgery, University of Southern California, Los Angeles, California 90033. email: tcchen@usc.edu. 